\title{
A study on the AH1N1/09 influenza transmission model with the fractional Caputo-Fabrizio derivative
}

\author{
Shahram Rezapour ${ }^{1,2,3}$ and Hakimeh Mohammadi ${ }^{*}$
}

\section{"Correspondence:}

hakimeh.mohammadi@yahoo.com

${ }^{4}$ Department of Mathematics,

Miandoab Branch, Islamic Azad

University, Miandoab, Iran

Full list of author information is

available at the end of the article

\section{Springer}

\begin{abstract}
We study the SEIR epidemic model for the spread of AH1N1 influenza using the Caputo-Fabrizio fractional-order derivative. The reproduction number of system and equilibrium points are calculated, and the stability of the disease-free equilibrium point is investigated. We prove the existence of solution for the model by using fixed point theory. Using the fractional Euler method, we get an approximate solution to the model. In the numerical section, we present a simulation to examine the system, in which we calculate equilibrium points of the system and examine the behavior of the resulting functions at the equilibrium points. By calculating the results of the model for different fractional order, we examine the effect of the derivative order on the behavior of the resulting functions and obtained numerical values. We also calculate the results of the integer-order model and examine their differences with the results of the fractional-order model.
\end{abstract}

MSC: Primary 34A08; secondary 65P99; 49J15

Keywords: AH1N1 influenza; Equilibrium point; Numerical simulation; SEIR model

\section{Introduction}

The pandemic virus AH1N1/09 that was identified in April 2009 is a flu virus of swine, avian, and human origin. This virus first was identified in Mexico and the USA and then spread to the rest of the world so that the WHO declared the new influenza $\mathrm{A}(\mathrm{H} 1 \mathrm{~N} 1)$ a pandemic on June 11, 2009 [1]. Through effective contacts of susceptible people with infectious people, the virus AH1N1 transmits. In the USA, approximately 36,000 people die from seasonal influenza or flu-related causes every year. Due to the importance of vaccination in epidemics, many attempts were made to find the vaccine of this disease until the first effective vaccine was found in the United States in October 2009 [2].

To investigate the dynamic behavior of epidemic diseases, mathematical models have an important role. There are several mathematical models such as SI, SIR, SIS, SIRS, and SEIR [3]. To study the dynamics of H1N1 influenza virus transmission, several mathematical models have been presented. The SIR model has been presented in the approach of Ebenezer [4], Hattaf et al. [5]. El-Shahed and his colleagues used the SIRC model [6] to investigate this disease transmission. Karim and Razali examined the spread of influenza in

(c) The Author(s) 2020. This article is licensed under a Creative Commons Attribution 4.0 International License, which permits use, sharing, adaptation, distribution and reproduction in any medium or format, as long as you give appropriate credit to the original author(s) and the source, provide a link to the Creative Commons licence, and indicate if changes were made. The images or other third party material in this article are included in the article's Creative Commons licence, unless indicated otherwise in a credit line to the material. If material is not included in the article's Creative Commons licence and your intended use is not permitted by statutory regulation or exceeds the permitted use, you will need to obtain permission directly from the copyright holder. To view a copy of this licence, visit http://creativecommons.org/licenses/by/4.0/ 
Malaysia using the SEIRS model [7]. Altaf Khan and his colleagues [8] and Gonzalez-Parra [9] also studied the spread of influenza using the mathematical SEIR model, which is one of the good models in the study of the spread of diseases. With the spread of influenza, Tan et al. used the SEIARC model [10], which has two groups more than the SEIR model and includes more details, to analyze the spread of the disease in Guangdong province. The study of diseases dynamics is a dominating theme for many biologists and mathematicians (see, for example, [11-21]).

It has been studied by many researchers that fractional extensions of mathematical models of integer order represent the natural fact in a very systematic way such as in the approach of Akbari et al. [22], Baleanu et al. [23-25], Kumar et al. [26], Singh et al. [27]. With the expansion of the application of fractional derivatives, methods for solving fractional mathematical systems have been considered by many researchers (see, for example, [28-35]). Also, the study of the mathematical model of phenomena with fractional order derivatives and their optimal control has been the subject of research by many researchers (see, for example, [36-40]). In recent years, many papers have been published on the subject of Caputo-Fabrizio fractional derivative (see, for example, [41-48]).

Given that research conducted in the recent decade shows that fractional-order derivatives work better in modeling real phenomena than integer-order derivatives and include the system of internal memory, in this paper, we study the mathematical model for AH1N1/09 influenza transmission [9] by using the Caputo-Fabrizio fractional derivative. In order to examine the difference between the results of the model with the fractional and integer order, in the numerical part, we also obtain the results with the integer derivative and compare them. We obtain the reproduction number and equilibrium points of the system, and in the numerical simulation we examine the behavior of the system at equilibrium points. In the integer-order derivative, the least change in the order is a unit and the effect of small changes in the derivative order in the results cannot be examined. In this work, we obtain the results of the fractional-order model for different values of derivative order and investigate the effect of derivation order on the results.

The structure of the paper is as follows. In Sect. 2 some basic definitions and concepts of fractional calculus are recalled. The SEIR model of fractional order for AH1N1/09 influenza transmission is presented in Sect. 3. In Sect. 4, the equilibrium points and the reproduction number are calculated and the stability of the equilibrium points is investigated. The existence of solution for the system is proved in Sect. 5. In Sect. 6, a numerical method for solving the model is described and a numerical simulation is presented.

\section{Preliminaries}

In this section, we recall some of the fundamental concepts of fractional differential calculus, which are found in many books and papers.

Definition 1 ([49]) For an integrable function $g$, the Caputo derivative of fractional order $v \in(0,1)$ is given by

$$
{ }^{C} D^{v} g(t)=\frac{1}{\Gamma(m-v)} \int_{0}^{t} \frac{g^{(m)}(v)}{(t-v)^{v-m+1}} d v, \quad m=[v]+1 .
$$


Also, the corresponding fractional integral of order $v$ with $\operatorname{Re}(v)>0$ is given by

$$
{ }^{C} I^{v} g(t)=\frac{1}{\Gamma(v)} \int_{0}^{t}(t-v)^{v-1} g(v) d v .
$$

Definition $2([41,50])$ For $g \in H^{1}(c, d)$ and $d>c$, the Caputo-Fabrizio derivative of fractional order $v \in(0,1)$ for $g$ is given by

$$
{ }^{C F} D^{v} g(t)=\frac{M(v)}{(1-v)} \int_{c}^{t} \exp \left(\frac{-v}{1-v}(t-v)\right) g^{\prime}(v) d v
$$

where $t \geq 0, M(v)$ is a normalization function that depends on $v$ and $M(0)=M(1)=1$. If $g \notin H^{1}(c, d)$ and $0<v<1$, this derivative for $g \in L^{1}(-\infty, d)$ is given by

$$
{ }^{C F} D^{v} g(t)=\frac{v M(v)}{(1-v)} \int_{-\infty}^{d}(g(t)-g(v)) \exp \left(\frac{-v}{1-v}(t-v)\right) d v .
$$

Also, the corresponding $C F$ fractional integral is presented by

$$
{ }^{C F} I^{v} g(t)=\frac{2(1-v)}{(2-v) M(v)} g(t)+\frac{2 v}{(2-v) M(v)} \int_{0}^{t} g(v) d v \text {. }
$$

The Laplace transform is one of the important tools in solving differential equations that are defined below for two kinds of fractional derivative.

Definition 3 ([49]) The Laplace transform of Caputo fractional differential operator of order $v$ is given by

$$
L\left[{ }^{C} D^{v} g(t)\right](s)=s^{\nu} L g(t)-\sum_{i=0}^{m-1} s^{\nu-i-1} g^{(i)}(0), \quad m-1<v \leq m \in N,
$$

which can also be obtained in the form

$$
L\left[{ }^{C} D^{v} g(t)\right]=\frac{s^{m} L[g(t)]-s^{m-1} g(0)-s^{m-1} g^{\prime}(0)-\cdots-g^{(m-1)}}{s^{m-v}} .
$$

Definition 4 ([41]) The Laplace transform of the Caputo-Fabrizio derivative is defined by

$$
L\left[{ }^{C F} D^{(\alpha)} f(t)\right](s)=\frac{M(\alpha)(2-\alpha)}{2(s+\alpha(1-s))}(s L[f(t)]-f(0)) .
$$

Since $M(\alpha)=\frac{2}{2-\alpha}$ for $0<\alpha<1$, we get

$$
L\left[{ }^{C F} D^{(\alpha)} f(t)\right](s)=\frac{s L[f(t)]-f(0)}{(s+\alpha(1-s))} .
$$

Definition 5 ([51]) Let $(X, d)$ be a metric space, a map $g: X \rightarrow X$ is called a Picard operator whenever there exists $x^{*} \in X$ such that $F i x(g)=\left\{x^{*}\right\}$ and the sequence $\left(g^{n}\left(x_{0}\right)\right)_{n \in N}$ converges to $x^{*}$ for all $x_{0} \in X$. 


\section{Mathematical model of the AH1N1/09 influenza transmission}

With the global outbreak of influenza AH1N1 virus in 2009, which killed more than 14,000 people worldwide, various mathematical models have been developed to study and simulate the spread of the virus. One of these models that have good results in epidemic diseases is the SEIR model, which has been modeled and studied by Gilberto GonzálezParra and his colleagues with the integer-order derivative [9]. Considering the good results of fractional derivative order in modeling real phenomena in recent years, in this work we investigate the SEIR model of AH1N1/09 influenza virus transmission with CaputoFabrizio fractional-order derivative.

In this model, the total population $N(t)$ is divided into four categories: the susceptible individuals $S(t)$, the exposed individuals $E(t)$, the infectious individuals $I(t)$, and the individuals who have recovered $R(t)$. The desired SEIR model is as follows:

$$
\left\{\begin{array}{l}
\frac{d S}{d t}=\kappa-\beta S(t) I(t)-m S(t), \\
\frac{d E}{d t}=\beta S(t) I(t)-(m+\delta) E(t), \\
\frac{d I}{d t}=\delta E(t)-(m+\mu) I(t) \\
\frac{d R}{d t}=\mu I(t)-m R(t)
\end{array}\right.
$$

where $\kappa$ : the birth rate of people, $m$ : the death rate of people, $\beta$ : the transmission rate of infection from $I$ to $S, \delta$ : the transmission rate of people from $E$ to $I, \mu$ : the recovery rate of infected people, with initial conditions $S(0)=S_{0}>0, E(0)=E_{0}>0, I(0)=I_{0}>0, R(0)=$ $R_{0} \geq 0$.

In this section, we moderate the system by substituting the time derivative by the Caputo-Fabrizio fractional derivative. With this change, the right- and left-hand sides will not have the same dimension. To solve this problem, we use an auxiliary parameter $\theta$, having the dimension of sec., to change the fractional operator so that the sides have the same dimension [52]. According to the explanation presented, the fractional model of the H1N1/09 influenza transmission for $t \geq 0$ and $v \in(0,1)$ is given as follows:

$$
\left\{\begin{array}{l}
\eta^{\nu-1 C F} D_{t}^{v} S(t)=\kappa-\beta S(t) I(t)-m S(t), \\
\eta^{\nu-1 C F} D_{t}^{v} E(t)=\beta S(t) I(t)-(m+\delta) E(t), \\
\eta^{\nu-1 C F} D_{t}^{v} I(t)=\delta E(t)-(m+\mu) I(t), \\
\eta^{\nu-1 C F} D_{t}^{v} R(t)=\mu I(t)-m R(t),
\end{array}\right.
$$

where the initial conditions are $S(0)=S_{0}>0, E(0)=E_{0}>0, I(0)=I_{0}>0, R(0)=R_{0} \geq 0$.

\section{Equilibrium points}

To determine the equilibrium points of fractional order system (1), we solve the following equations:

$$
{ }^{C F} D^{v} S(t)={ }^{C F} D^{v} E(t)={ }^{C F} D^{v} I(t)={ }^{C F} D^{v} R(t)=0 .
$$

By solving the algebraic equations, we obtain equilibrium points of system (1). The disease-free equilibrium point is obtained as $E_{0}=\left(\frac{\kappa}{m}, 0,0,0\right)$, and if $R_{0}>1$ then the sys- 
tem has the endemic equilibrium point $E_{1}=\left(S^{*}, E^{*}, I^{*}, R^{*}\right)$ so that

$$
\begin{aligned}
& S^{*}=\frac{\delta(m+\mu)+m^{2}+m \mu}{\beta \delta}, \\
& E^{*}=\frac{\beta \delta \kappa-\delta m^{2}-\delta m \mu-m^{3}-m^{2} \mu}{\delta \beta(m+\delta)}, \\
& I^{*}=\frac{\beta \delta \kappa-\delta m^{2}-\delta m \mu-m^{3}-m^{2} \mu}{\beta\left(\delta m+\delta \mu+m^{2}+m \mu\right)}, \\
& R^{*}=\frac{\mu\left(\beta \delta \kappa-\delta m^{2}-\delta m \mu-m^{3}-m^{2} \mu\right)}{\beta m\left(\delta m+\delta \mu+m^{2}+m \mu\right)} .
\end{aligned}
$$

Also, $R_{0}$ is the basic reproduction number and is obtained using the next generation method [53]. To find $R_{0}$, we first consider the system as follows:

$$
{ }^{C} D^{v} \Psi(t)=F(\Psi(t))-V(\Psi(t))
$$

where

$$
F(\Psi(t))=\eta^{1-\nu}\left[\begin{array}{c}
\beta S(t) I(t) \\
0
\end{array}\right]
$$

and

$$
V(\Psi(t))=\eta^{1-v}\left[\begin{array}{c}
(m+\delta) E(t) \\
-\delta E(t)+(m+\mu) I(t)
\end{array}\right] .
$$

At $E^{0}$, the Jacobian matrix for $F$ and $V$ is obtained as follows:

$$
J_{F}\left(E_{0}\right)=\eta^{1-v}\left[\begin{array}{cc}
0 & \beta \frac{\kappa}{m} \\
0 & 0
\end{array}\right], \quad J_{v}\left(E_{0}\right)=\eta^{1-v}\left[\begin{array}{cc}
m+\delta & 0 \\
-\delta & m+\mu
\end{array}\right] .
$$

$F V^{-1}$ is the next generation matrix for system (1), and the basic reproduction number is obtained from $R_{0}=\rho\left(F V^{-1}\right)$. So we obtain the reproduction as $R_{0}=\frac{\beta \delta \kappa}{m}$. This basic reproduction number $R_{0}$ is an epidemiologic metric used to describe the contagiousness or transmissibility of infectious agents.

\subsection{Stability of equilibrium point}

To investigate the stability of an equilibrium point, first consider the fractional-order linear system

$$
{ }^{C F} D_{t}^{v} y(t)=T y(t)
$$

where $y(t) \in R^{n}, T \in R^{n \times n}, 0<v<1$.

Definition 6 ([54]) For system (2) with Caputo-Fabrizio fractional derivative, the characteristic equation is given by

$$
\operatorname{det}(s(I-(1-v) T)-v T)=0 .
$$


Theorem 7 ([54]) If $(I-(1-v) T)$ is invertible, then system (2) is asymptotically stable if and only if the roots to the characteristic equation of system (3) have negative real parts.

The Jacobian matrix associated with system (2) is given as follows:

$$
J=\eta^{1-v}\left[\begin{array}{cccc}
-(\beta I+m) & 0 & -\beta S & 0 \\
\beta I & -(m+\delta) & \beta S & 0 \\
0 & \delta & -(m+\mu) & 0 \\
0 & 0 & \mu & -m
\end{array}\right]
$$

Then the Jacobian matrix at $E_{0}$ is

$$
J\left(E_{0}\right)=\eta^{1-\nu}\left[\begin{array}{cccc}
-m & 0 & -\beta \frac{\kappa}{m} & 0 \\
0 & -(m+\delta) & \beta \frac{\kappa}{m} & 0 \\
0 & \delta & -(m+\mu) & 0 \\
0 & 0 & \mu & -m
\end{array}\right] .
$$

The characteristic equation of $J\left(E_{0}\right)$ is

$$
\begin{aligned}
& {[s(1+m(1-v))+m v]^{2}[s(1+(1-v)(m+\delta))+v(m+\delta)]} \\
& \quad \times[s(1+(1-v)(m+\mu))+v(m+\mu)]=0 .
\end{aligned}
$$

By computing the roots of the above equation, we obtain

$$
\begin{aligned}
& s_{1}=s_{2}=\frac{-m v}{1+m(1-v)}, \\
& s_{3}=\frac{-v(m+\delta)}{1+(1-v)(m+\delta)}, \\
& s_{4}=\frac{-v(m+\mu)}{1+(1-v)(m+\mu)} .
\end{aligned}
$$

Since all of the parameters are positive and $0<v<1$, then the roots of characteristic equation are negative. Thus by using Theorem 7 , the disease-free equilibrium point $E_{0}$ of model (1) is asymptotically stable.

\section{Existence of solution}

The system of differential equations for the AH1N1 disease model (1) using the CaputoFabrizio fractional-order derivative is considered as follows:

$$
\left\{\begin{array}{l}
{ }^{C F} D_{t}^{v} S(t)=\eta^{1-v}[\kappa-\beta S(t) I(t)-m S(t)], \\
{ }^{C F} D_{t}^{v} E(t)=\eta^{1-v}[\beta S(t) I(t)-(m+\delta) E(t)], \\
{ }^{C F} D_{t}^{v} I(t)=\eta^{1-v}[\delta E(t)-(m+\mu) I(t)], \\
{ }^{C F} D_{t}^{v} R(t)=\eta^{1-v}[\mu I(t)-m R(t)] .
\end{array}\right.
$$


Applying the Losada and Nieto integral operator [41] on both sides of equations (4), we obtain

$$
\begin{aligned}
S(t)-u_{1}(t)= & \eta^{1-v}\left[\frac{2(1-v)}{(2-v) M(v)}\{\kappa-\beta S(t) I(t)-m S(t)\}\right. \\
& \left.+\frac{2 v}{(2-v) M(v)} \int_{0}^{t}[\kappa-\beta S(\tau) I(\tau)-m S(\tau)] d \tau\right], \\
E(t)-u_{2}(t)= & \eta^{1-v}\left[\frac{2(1-v)}{(2-v) M(v)}\{\beta S(t) I(t)-(m+\delta) E(t)\}\right. \\
& \left.+\frac{2 v}{(2-v) M(v)} \int_{0}^{t}[\beta S(\tau) I(\tau)-(m+\delta) E(\tau)] d \tau\right], \\
I(t)-u_{3}(t)= & \eta^{1-v}\left[\frac{2(1-v)}{(2-v) M(v)}\{\delta E(t)-(m+\mu) I(t)\}\right. \\
& \left.+\frac{2 v}{(2-v) M(v)} \int_{0}^{t}[\delta E(\tau)-(m+\mu) I(\tau)] d \tau\right], \\
R(t)-u_{4}(t)= & \eta^{1-v}\left[\frac{2(1-v)}{(2-v) M(v)}\{\mu I(t)-m R(t)\}\right. \\
& \left.+\frac{2 v}{(2-v) M(v)} \int_{0}^{t}[\mu I(\tau)-m R(\tau)] d \tau\right] .
\end{aligned}
$$

We present the differential equations (5) as follows:

$$
\begin{aligned}
& S_{0}(t)=u_{1}(t), \quad E_{0}(t)=u_{2}(t), \\
& I_{0}(t)=u_{3}(t), \quad R_{0}(t)=u_{4}(t), \\
& S_{n+1}(t)= \eta^{1-v}\left[\frac{2(1-v)}{(2-v) M(v)}\{\kappa-\beta S(t) I(t)-m S(t)\}\right. \\
&\left.+\frac{2 v}{(2-v) M(v)} \int_{0}^{t}[\kappa-\beta S(\tau) I(\tau)-m S(\tau)] d \tau\right], \\
&\left.E_{n+1}(t)\right)= \eta^{1-v}\left[\frac{2(1-v)}{(2-v) M(v)}\{\beta S(t) I(t)-(m+\delta) E(t)\}\right. \\
&\left.+\frac{2 v}{(2-v) M(v)} \int_{0}^{t}[\beta S(\tau) I(\tau)-(m+\delta) E(\tau)] d \tau\right], \\
& I_{n+1}(t)= \eta^{1-v}\left[\frac{2(1-v)}{(2-v) M(v)}\{\delta E(t)-(m+\mu) I(t)\}\right. \\
&\left.+\frac{2 v}{(2-v) M(v)} \int_{0}^{t}[\delta E(\tau)-(m+\mu) I(\tau)] d \tau\right], \\
& R_{n+1}(t)= \eta^{1-v}\left[\frac{2(1-v)}{(2-v) M(v)}\{\mu I(t)-m R(t)\}\right. \\
&\left.+\frac{2 v}{(2-v) M(v)} \int_{0}^{t}[\mu I(\tau)-m R(\tau)] d \tau\right] .
\end{aligned}
$$


By taking the limit from above Picard's repetitive series when $n$ tends to $\infty$, we obtain the solution of the equation as follows:

$$
\left\{\begin{array}{l}
\lim _{n \rightarrow \infty} S_{n}(t)=S(t), \\
\lim _{n \rightarrow \infty} E_{n}(t)=E(t), \\
\lim _{n \rightarrow \infty} I_{n}(t)=I(t), \\
\lim _{n \rightarrow \infty} R_{n}(t)=R(t) .
\end{array}\right.
$$

\subsection{Existence of solution by the Picard-Lindelof approach}

Using the Picard-Lindelof approach and the Banach fixed point theorem, we prove the existence of solution. We define the following operators:

$$
\left\{\begin{array}{l}
h_{1}(t, S)=\eta^{1-v}[\kappa-\beta S(t) I(t)-m S(t)], \\
h_{2}(t, E)=\eta^{1-v}[\beta S(t) I(t)-(m+\delta) E(t)], \\
h_{3}(t, I)=\eta^{1-v}[\delta E(t)-(m+\mu) I(t)], \\
h_{4}(t, R)=\eta^{1-v}[\mu I(t)-m R(t)] .
\end{array}\right.
$$

Let $L_{1}=\sup _{C\left[a, c_{1}\right]}\left\|h_{1}(t, S)\right\|, L_{2}=\sup _{C\left[a, c_{2}\right]}\left\|h_{2}(t, E)\right\|, L_{3}=\sup _{C\left[a, c_{3}\right]}\left\|h_{3}(t, I)\right\|$, and $L_{4}=$ $\sup _{C\left[a, c_{4}\right]}\left\|h_{4}(t, R)\right\|$, where

$$
\left\{\begin{array}{l}
C\left[a, c_{1}\right]=|t-a, t+a| \times\left|S-c_{1}, S+c_{1}\right|=A \times C_{1}, \\
C\left[a, c_{2}\right]=|t-a, t+a| \times\left|E-c_{2}, E+c_{2}\right|=A \times C_{2}, \\
C\left[a, c_{3}\right]=|t-a, t+a| \times\left|I-c_{3}, I+c_{3}\right|=A \times C_{3}, \\
C\left[a, c_{4}\right]=|t-a, t+a| \times\left|R-c_{4}, R+c_{4}\right|=A \times C_{4} .
\end{array}\right.
$$

Now, we assume a uniform norm on $C\left[a, c_{i}\right],(i=1,2,3,4)$ as follows:

$$
\|Y(t)\|_{\infty}=\sup _{t \in[t-a, t+a]}|Y(t)| .
$$

Consider the Picard operator

$$
\Theta: C\left(A, C_{1}, C_{2}, C_{3}, C_{4}\right) \rightarrow C\left(A, C_{1}, C_{2}, C_{3}, C_{4}\right)
$$

given as follows:

$$
\Theta(Y(t))=Y_{0}(t)+\frac{2(1-v)}{2-v) M(v)} H(t, Y(t))+\frac{2 v}{(2-v) M(v)} \int_{0}^{t} H(\tau, Y(\tau)) d \tau
$$

so that $Y(t)=\{S(t), E(t), I(t), R(t)\}, Y_{0}(t)=\{S(0), E(0), I(0), R(0)\}$ and

$$
H(t, Y(t))=\left\{h_{1}(t, S), h_{2}(t, E), h_{3}(t, I), h_{4}(t, R)\right\}
$$

We assume that the solutions of system (1) are bounded within a time period,

$$
\|Y(t)\|_{\infty} \leq \max \left\{c_{1}, c_{2}, c_{3}, c_{4}\right\}=C
$$


Let $L=\max \left\{L_{1}, L_{2}, L_{3}, L_{4}\right\}$ and there exist $t_{0}$ so that $t_{0} \geq t$, then

$$
\begin{aligned}
\| \Theta & Y(t)-Y_{0}(t) \| \\
& =\left\|\frac{2(1-v)}{(2-v) M(v)} H(t, Y(t))+\frac{2 v}{(2-v) M(v)} \int_{0}^{t} H(\tau, Y(\tau)) d \tau\right\|, \\
& \leq \frac{2(1-v)}{(2-v) M(v)}\|H(t, Y)\|+\frac{2 v}{(2-v) M(v)} \int_{0}^{t}\|H(\tau, Y)\| d \tau, \\
& \leq\left(\frac{2(1-v)}{(2-v) M(v)}+\frac{2 v t}{(2-v) M(v)}\right) L \\
& \leq\left(\frac{2(1-v)}{(2-v) M(v)}+\frac{2 v t_{0}}{(2-v) M(v)}\right) L \leq \gamma L \leq C,
\end{aligned}
$$

where we demand that

$$
\gamma<\frac{C}{L} .
$$

Also we evaluate the following equality:

$$
\left\|\Theta Y_{1}-\Theta Y_{2}\right\|=\sup _{t \in A}\left|Y_{1}(t)-Y_{2}(t)\right|
$$

By our Picard's operator, we obtain

$$
\begin{aligned}
\left\|\Theta Y_{1}-\Theta Y_{2}\right\|= & \| \frac{2(1-v)}{(2-v) M(v)}\left\{H \left(t, Y_{1}(t)-H\left(t, Y_{2}(t)\right\}\right.\right. \\
& +\frac{2 v}{(2-v) M(v)} \int_{0}^{t}\left\{H \left(\tau, Y_{1}(\tau)-H\left(\tau, Y_{2}(\tau)\right\} d \tau \|,\right.\right. \\
\leq & \frac{2(1-v)}{(2-v) M(v)} \| H\left(t, Y_{1}(t)-H\left(t, Y_{2}(t) \|\right.\right. \\
& +\frac{2 v}{(2-v) M(v)} \int_{0}^{t} \| H\left(\tau, Y_{1}(\tau)-H\left(\tau, Y_{2}(\tau) \| d \tau,\right.\right. \\
\leq & \frac{2(1-v)}{(2-v) M(v)} \rho\left\|Y_{1}(t)-Y_{2}(t)\right\| \\
& +\frac{2 v \rho}{(2-v) M(v)} \int_{0}^{t}\left\|Y_{1}(\tau)-Y_{2}(\tau)\right\| d \tau, \\
\leq & \left(\frac{2(1-v) \lambda}{(2-v) M(v)}+\frac{2 v \rho t_{0}}{(2-v) M(v)}\right)\left\|Y_{1}(t)-Y_{2}(t)\right\| \\
\leq & \gamma \rho\left\|Y_{1}(t)-Y_{2}(t)\right\|
\end{aligned}
$$

with $\rho<1$. Since $H$ is a contraction, then $\gamma \rho<1$, this proves that $\Theta$ is a contraction and completes the proof.

\section{Numerical results}

Using the fractional Euler method for Caputo-Fabrizio derivative, we present the approximate solutions for a fractional-order SEIR model of the AH1N1/09 influenza transmission model. 


\subsection{Numerical method}

We consider system (1) in a compact form as follows:

$$
\eta^{\nu-1 C} D_{t}^{\nu} w(t)=g(t, w(t)), \quad w(0)=w_{0}, \quad 0 \leq t \leq T<\infty,
$$

where $w=(S, E, I, R) \in R_{+}^{4}, w_{0}=\left(S_{0}, E_{0}, I_{0}, R_{0}\right)$ is the initial vector, and $g(t) \in R$ is a continuous vector function satisfying the Lipschitz condition

$$
\left\|g\left(w_{1}(t)\right)-g\left(w_{2}(t)\right)\right\| \leq k\left\|w_{1}(t)-w_{2}(t)\right\|, \quad k>0 .
$$

Applying a fractional integral operator corresponding to Caputo derivative to equation (7), we obtain

$$
w(t)=\eta^{1-\nu}\left[w_{0}+I^{v} g(w(t))\right], \quad 0 \leq t \leq T<\infty .
$$

Set $h=\frac{T-0}{N}$ and $t_{n}=n h$, where $t \in[0, T]$ and $N$ is a natural number and $n=0,1,2, \ldots, N$. Let $w_{n}$ be the approximation of $w(t)$ at $t=t_{n}$. Using the fractional Euler method [55, 56], we get

$$
w_{n+1}=\eta^{1-v}\left[w_{0}+(1-v) g\left(t_{n+1}, w_{n+1}\right)+v h \sum_{j=0}^{n} g\left(t_{j}, w_{j}\right)\right], \quad n=0,1,2, \ldots, N-1,
$$

the stability analysis of the obtained scheme has been proved in Theorem (3.1) in [55].

Thus, the solution of system (1) is written as follows:

$$
\begin{aligned}
& S_{n+1}=\eta^{1-v}\left[S_{0}+(1-v) f_{1}\left(t_{n+1}, w_{n+1}\right)+v h \sum_{j=0}^{n} f_{1}\left(t_{j}, w_{j}\right)\right], \\
& E_{n+1}=\eta^{1-v}\left[E_{0}+(1-v) f_{2}\left(t_{n+1}, w_{n+1}\right)+v h \sum_{j=0}^{n} f_{2}\left(t_{j}, w_{j}\right)\right], \\
& I_{n+1}=\eta^{1-v}\left[I_{0}+(1-v) f_{3}\left(t_{n+1}, w_{n+1}\right)+v h \sum_{j=0}^{n} f_{3}\left(t_{j}, w_{j}\right)\right], \\
& R_{n+1}=\eta^{1-v}\left[R_{0}+(1-v) f_{4}\left(t_{n+1}, w_{n+1}\right)+v h \sum_{j=0}^{n} f_{4}\left(t_{j}, w_{j}\right)\right],
\end{aligned}
$$

where $f_{1}(t, w(t))=\kappa-\beta S(t) I(t)-m S(t), f_{2}(t, w(t))=\beta S(t) I(t)-(m+\delta) E(t), f_{3}(t, w(t))=$ $\delta E(t)-(m+\mu) I(t), f_{4}(t, w(t))=\mu I(t)-m R(t)$.

\subsection{Numerical simulation}

To check the behavior of the model, we use the parameters obtained by Gonzalez-Parra et al. [9]. The reported incubation period for the AH1N1/09 virus is $2--10$ days. So the assumed mean time in $E(t)$ is $\delta=\frac{1}{5}$ days $^{-1}$. The reported infectious period is 4-7 days, so it has been assumed $\mu=\frac{1}{7}$. Since the used time period is short, then the population size is assumed to be constant, and thus $\kappa=m=\frac{0.015}{52}$ days $^{-1}$. Also, by fitting the data technique 


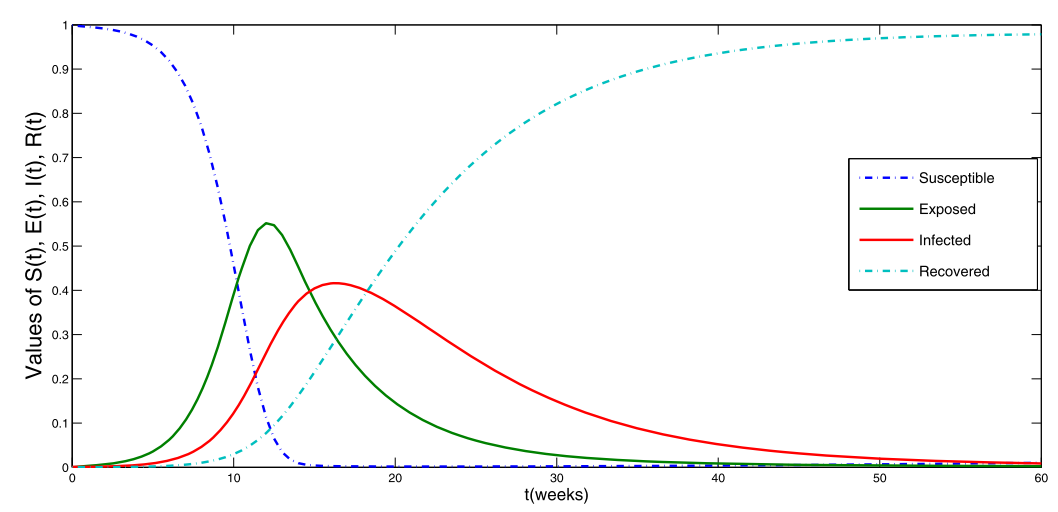

Figure 1 Dynamics of $S(t), E(t), I(t), R(t)$ for $v=0.98$
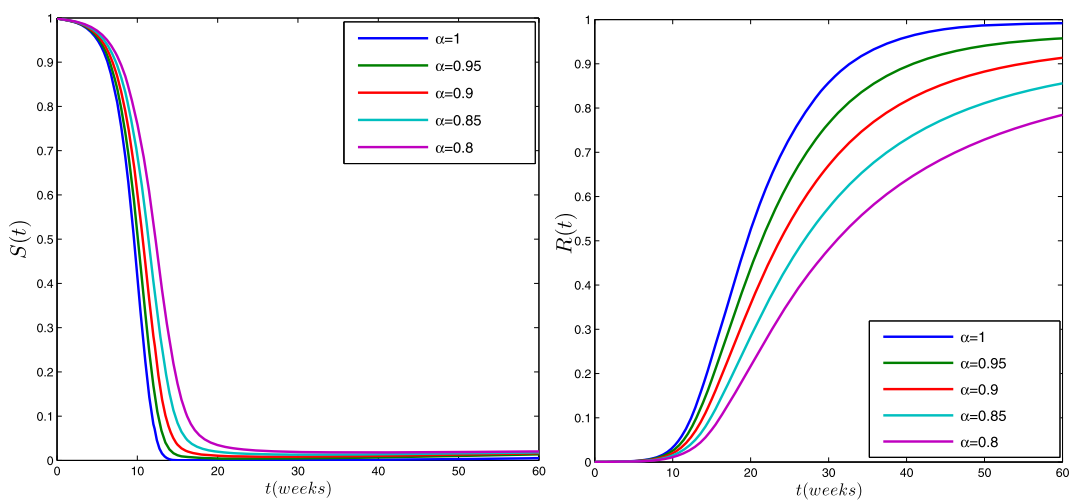

Figure 2 Dynamics of $S(t)$ and $E(t)$ for different values of $v=1,0.95,0.9,0.85,0.8$

and confirmed cases of pandemic AH1N1/09 influenza for Bogota D.C, it is obtained $\beta=$ 3.58. Also, the initial conditions are $S(0)=1-0.001, E(0)=0, I(0)=0.001, R(0)=0$.

In this simulation, the reproduction number is $R_{0}=0.716$ and the endemic equilibrium point is

$$
E_{1}=\left(S^{*}, E^{*}, I^{*}, R^{*}\right)=(0.04004248,0.00138256,0.00193168,0.95664328) .
$$

In Fig. 1, the answers of the fractional-order model for AH1N1 influenza with $v=0.98$ are plotted. In this simulation, the value of $R_{0}$ is equal to 0.716 which is smaller than 1 and, as you can see, the spread of the disease is controlled and the number of infected people is reduced to zero. We also see that each of the functions tends to its equilibrium point and the system in equilibrium points becomes stable. In Figs. 2 and 3, we have plotted the results of model (1) for different fractional orders $v=1,0.95,0.9,0.85,0.8$. Given that disease propagation models are usually used in predicting disease progression and making controlling decisions, it is important to determine the exact order of derivation of the model, as shown in Fig. 3, while in model 1 after the disease is controlled for 60 days. But in the model, with the order of 0.9 or 0.8 the disease still persists and it takes several days to be controlled. The results of the AH1N1 influenza transmission model (1) are plotted for 

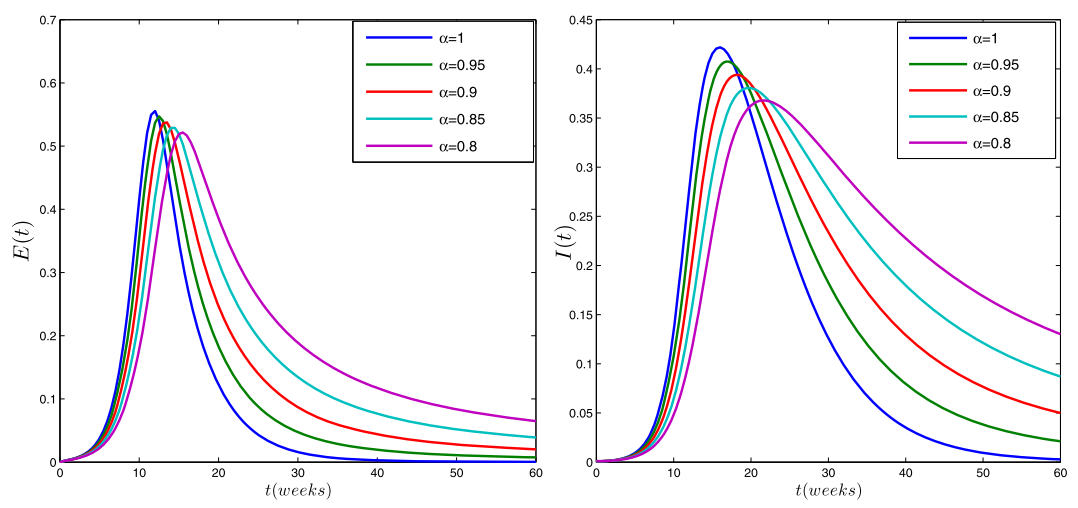

Figure 3 Dynamics of $S(t)$ and $E(t)$ for different values of $v=1,0.95,0.9,0.85,0.8$
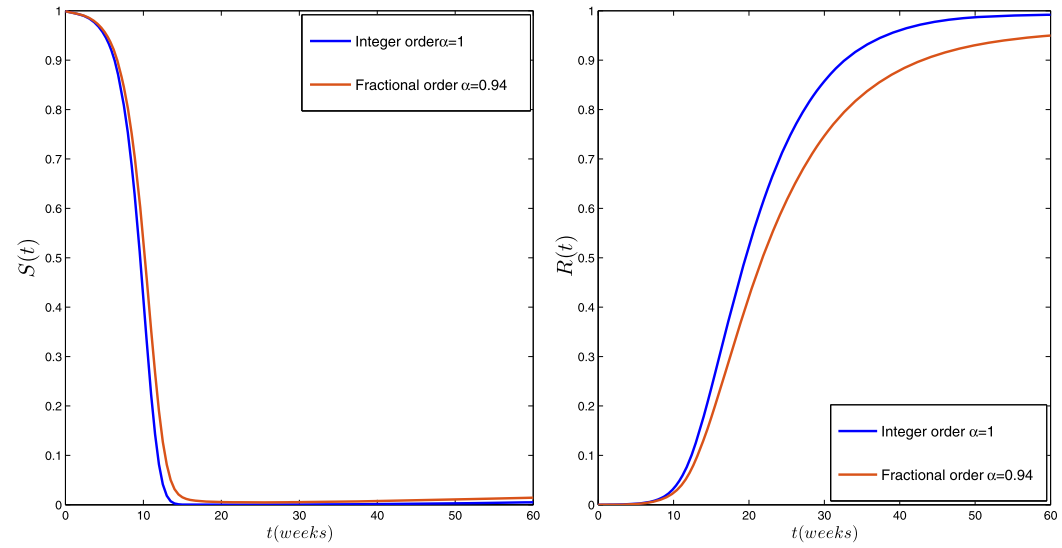

Figure 4 Plots of $S(t)$ and $R(t)$ for integer order $v=1$ and fractional order $v=0.94$

fractional-order Caputo-Fabrizio derivatives $v=0.94$ and integer-order derivative $v=1$ in Figs. 4-5. Comparison of the graphs shows that the resulting values are different, but the behavior of the functions derived from both types of derivatives is the same.

\section{Conclusion}

In this work, the SEIR epidemic model for the transmission of AH1N1 influenza using the Caputo-Fabrizio fractional-order derivative has been presented. The reproduction number of the system and equilibrium points have been calculated and the stability of a disease-free equilibrium point has been investigated. The existence of solution for the model by using fixed point theory has been proved. Using the fractional Euler method, an approximate answer to the model has been calculated. Also, with a numerical simulation, the values of reproduction number and equilibrium points are calculated, and the results show that the system is stable at equilibrium points and each of the obtained functions converges to its equilibrium point. According to the obtained reproduction number $R_{0}=0.716<1$, the epidemic has been controlled and the number of infected people has reduced to zero. To investigate the effect of derivative order on the model results, the functions obtained from the model are plotted for different degrees of fraction, and the results show that the general behavior of the functions is the same in small changes of derivative 

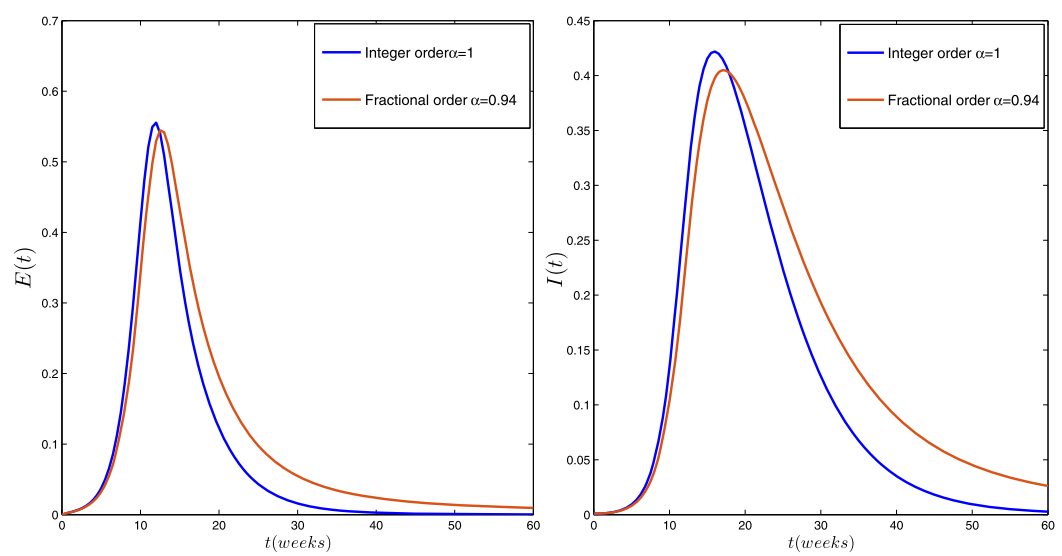

Figure 5 Plots of $E(t)$ and $/(t)$ for integer order $v=1$ and fractional order $v=0.94$

order but the numerical results are different. In future studies, the effect of each of the coefficients in Model 1 on the disease transmission process can be investigated. Also, research can be done to optimally control the disease spread model and the effect of drugs and vaccination on the current model.

\section{Acknowledgements}

The first author was supported by Azarbaijan Shahid Madani University. The second author was supported by Miandoab Branch, Islamic Azad University. The authors express their gratitude to dear unknown referees for their helpful suggestions which improved the final version of this paper.

Funding

Not applicable.

Availability of data and materials

Data sharing not applicable to this article as no datasets were generated or analyzed during the current study.

Ethics approval and consent to participate

Not applicable.

Competing interests

The authors declare that they have no competing interests.

Consent for publication

Not applicable.

Authors' contributions

The authors declare that the study was realized in collaboration with equal responsibility. All authors read and approved the final manuscript.

\section{Authors' information}

Shahram Rezapour: shahramrezapour@duytan.edu.vn,sh.rezapour@mail.cmuh.org.tw,sh.rezapour@azaruniv.ac.ir, rezapourshahram@yahoo.ca

\section{Author details}

${ }^{1}$ Institute of Research and Development, Duy Tan University, 550000, Da Nang, Vietnam. ${ }^{2}$ Faculty of Natural Sciences, Duy Tan University, 550000, Da Nang, Vietnam. ${ }^{3}$ Department of Medical Research, China Medical University Hospital, China Medical University, Taichung, Taiwan. ${ }^{4}$ Department of Mathematics, Miandoab Branch, Islamic Azad University, Miandoab, Iran.

\section{Publisher's Note}

Springer Nature remains neutral with regard to jurisdictional claims in published maps and institutional affiliations. 


\section{References}

1. WHO: 2009 H1N1 Flu. Centers for Disease Control and Prevention, http://www.cdc.gov/h1n1flu/ (2009)

2. Tracht, S., Valle, S.D., Hyman, J.: Mathematical modeling of the effectiveness of facemasks in reducing the spread of novel influenza A (H1N1). PLoS ONE 5(2), 9018 (2010)

3. Hethcote, H.: Mathematics of infectious diseases. SIAM Rev. 42(4), 599-653 (2005)

4. Ebenezer, B.: On fractional order influenza A epidemic model. Appl. Comput. Math. 4(2), 77-82 (2015)

5. Hattaf, K., Yousfi, N.: Mathematical model of influenza AH1N1 infection. Adv. Stud. Biol. 1(8), 383-390 (2009)

6. El-Shahed, M., Alsaedi, A.: The fractional SIRC model and influenza A. Math. Probl. Eng. 2011, 480378 (2011)

7. Karim, S.A.A., Razali, R.: A proposed mathematical model of influenza AH1N1 for Malaysia. J. Appl. Sci. 11(8), $1457-1460$ (2011)

8. Khan, M.A., Ullah, S., Ullah, S., Farhan, M.: Fractional order SEIR model with generalized incidence rate. AIMS Math. 5(4), 2843-2857 (2020)

9. Gonzalez-Parra, G., Arenas, A.J., Aranda, D.F., Segovia, L.: Modeling the epidemic waves of AH1N1/09 influenza around the world. Spat. Spatio-Tempor. Epidemiol. 2(4), 219-226 (2011)

10. Tan, X., Yuan, L., Zhou, J., Zheng, Y., Yang, F.: Modeling the initial transmission dynamics of influenza AH1N1 in Guangdong province, China. Int. J. Infect. Dis. 17, 479-484 (2013)

11. Haq, F., Shah, K., Rahman, G., Shahzad, M.: Numerical analysis of fractional order model of HIV-1 infection of cd4+ t-cells. Comput. Methods Differ. Equ. 5(1), 1-11 (2017)

12. Koca, I.: Analysis of rubella disease model with non-local and non-singular fractional derivatives. J. Theories Appl. 8(1), 17-25 (2018)

13. Rida, S.Z., Arafa, A.A.M., Gaber, Y.A.: Solution of the fractional epidemic model by l-adm. J. Fract. Calc. Appl. 7(1), 189-195 (2016)

14. Singh, H., Dhar, J., Bhatti, H.S., Chandok, S.: An epidemic model of childhood disease dynamics with maturation delay and latent period of infection. Model. Earth Syst. Environ. 2, 79 (2016)

15. Tchuenche, J.M., Dube, N., Bhunu, C.P., Smith, R.J., Bauch, C.T.: The impact of media coverage on the transmission dynamics of human influenza. BMC Public Health 11, 1-5 (2011)

16. Upadhyay, R.K., Roy, P.: Spread of a disease and its effect on population dynamics in an eco-epidemiological system. Commun. Nonlinear Sci. Numer. Simul. 19(12), 4170-4184 (2014)

17. Baleanu, D., Aydogan, S.M., Mohammadi, H., Rezapour, S.: On modelling of epidemic childhood diseases with the Caputo-Fabrizio derivative by using the Laplace Adomian decomposition method. Alex. Eng. J. (2020). https://doi.org/10.1016/j.aej.2020.05.007

18. Baleanu, D., Rezapour, S., Mohammadi, H.: Some existence results on nonlinear fractional differential equations. Philos. Trans. R. Soc. Lond. Ser. A 2013, 371 (2013). https://doi.org/10.1098/rsta.2012.0144

19. Etemad, S., Rezapour, S., Samei, M.E.: On a fractional Caputo-Hadamard inclusion problem with sum boundary value conditions by using approximate endpoint property. Math. Model. Appl. Sci. (2020). https://doi.org/10.1002/mma.6644

20. Tuan, N.H., Mohammadi, H., Rezapour, S.: A mathematical model for COVID-19 transmission by using the Caputo fractional derivative. Chaos Solitons Fractals 140, 110107 (2020). https://doi.org/10.1016/j.chaos.2020.110107

21. Baleanu, D., Jajarmi, A., Mohammadi, H., Rezapour, S.: A new study on the mathematical modelling of human liver with Caputo-Fabrizio fractional derivative. Chaos Solitons Fractals 134, 109705 (2020)

22. Kojabad, E.A., Rezapour, S.: Approximate solutions of a sum-type fractional integro-differential equation by using Chebyshev and Legendre polynomials. Adv. Differ. Equ. 2017, 351 (2017)

23. Baleanu, D., Agarwal, R.P., Mohammadi, H., Rezapour, S.: Some existence results for a nonlinear fractional differential equation on partially ordered Banach spaces. Bound. Value Probl. 2013, 112 (2013)

24. Baleanu, D., Mohammadi, H., Rezapour, S.: Some existence results on nonlinear fractional differential equations. Philos. Trans. R. Soc. 2013, 371 (2013)

25. Baleanu, D., Mohammadi, H., Rezapour, S.: Analysis of the model of HIV-1 infection of CD4+ T-cell with a new approach of fractional derivative. Adv. Differ. Equ. 2020, 71 (2020)

26. Kumar, D., Singh, J., Baleanu, D.: On the analysis of vibration equation involving a fractional derivative with Mittag-Leffler law. Math. Methods Appl. Sci. 43(1), 443-457 (2020)

27. Singh, J., Kumar, D., Baleanu, D.: A new analysis of fractional fish farm model associated with Mittag-Leffler type kernel. Int. J. Biomath. 13(02), 2050010 (2020)

28. Jagdev, S., Adem, K., Devendra, K., Ram, S., Fadzilah, A.: Numerical study for fractional model of nonlinear predator-prey biological population dynamical system. Therm. Sci. 23(6), 2017-2025 (2019)

29. Singh, J., Kumar, D., Baleanu, D., Rathore, S.: An efficient numerical algorithm for the fractional Drinfeld-Sokolov-Wilson equation. Appl. Math. Comput. 335, 12-24 (2016)

30. Baleanu, D., Etemad, S., Rezapour, S.: On a fractional hybrid integro-differential equation with mixed hybrid integral boundary value conditions by using three operators. Alex. Eng. J. (2020). https://doi.org/10.1016/j.aej.2020.04.053

31. Agarwal, R.P., Baleanu, D., Hedayati, V., Rezapour, S.: Two fractional derivative inclusion problems via integral boundary conditions. Appl. Math. Comput. 257, 205-212 (2015). https://doi.org/10.1016/j.amc.2014.10.082

32. Alsaedi, A., Baleanu, D., Etemad, S., Rezapour, S.: On coupled systems of time-fractional differential problems by using a new fractional derivative. J. Funct. Spaces 2016, Article ID 4626940 (2016). https://doi.org/10.1155/2016/4626940

33. Baleanu, D., Hedayati, V., Rezapour, S.: On two fractional differential inclusions. SpringerPlus 5, 882 (2016). https://doi.org/10.1186/s40064-016-2564-z

34. Rezapour, S., Samei, M.E.: On the existence of solutions for a multi-singular pointwise defined fractional q-integro-differential equation. Bound. Value Probl. 2020, 38 (2020). https://doi.org/10.1186/s13661-020-01342-3

35. Samei, M.E., Rezapour, S.: On a system of fractional q-differential inclusions via sum of two multi-term functions on a time scale. Bound. Value Probl. 2020, 135 (2020). https://doi.org/10.1186/s13661-020-01433-1

36. Baleanu, D., Jajarmi, A., Sajjadi, S.S., Asad, J.H.: The fractional features of a harmonic oscillator with position-dependent mass. Commun. Theor. Phys. 72(5), 055002 (2020)

37. Sajjadi, S.S., Baleanu, D., Jajarmi, A., Pirouz, H.M.: A new adaptive synchronization and hyperchaos control of a biological snap oscillator. Chaos Solitons Fractals 138, 109919 (2020) 
38. Jajarmi, A., Yusuf, A., Baleanu, D., Inc, M.: A new fractional HRSV model and its optimal control: a non-singular operator approach. Phys. A, Stat. Mech. Appl. 547, 123860 (2020)

39. Yildiz, T.A., Jajarmi, A., Yildiz, B., Baleanu, D.: New aspects of time fractional optimal control problems within operators with nonsingular kernel. Discrete Contin. Dyn. Syst., Ser. S 13(3), 407-428 (2020)

40. Jajarmi, A., Baleanu, D.: On the fractional optimal control problems with a general derivative operator. Asian J. Control (2019). https://doi.org/10.1002/asjc.2282

41. Losada, J., Nieto, J.J.: Properties of the new fractional derivative without singular kernel. Prog. Fract. Differ. Appl. 1(2), 87-92 (2015)

42. Aydogan, S.M., Baleanu, D., Mousalou, A., Rezapour, S.: On high order fractional integro-differential equations including the Caputo-Fabrizio derivative. Bound. Value Probl. 2018, 90 (2018)

43. Dokuyucu, M.A., Celik, E., Bulut, H., Baskonus, H.M.: Cancer treatment model with the Caputo-Fabrizio fractional derivative. Eur. Phys. J. Plus 133, 92 (2018)

44. Khan, M.A., Hammouch, Z., Baleanu, D.: Modeling the dynamics of hepatitis E via the Caputo-Fabrizio derivative. Math. Model. Nat. Phenom. 14(3), 311 (2019)

45. Baleanu, D., Rezapour, S., Saberpour, Z.: On fractional integro-differential inclusions via the extended fractional Caputo-Fabrizio derivation. Bound. Value Probl. 2019, 79 (2019)

46. Baleanu, D., Mousalou, A., Rezapour, S.: On the existence of solutions for some infinite coefficient-symmetric Caputo-Fabrizio fractional integro-differential equations. Bound. Value Probl. 2017(1), 145 (2017). https://doi.org/10.1186/s13661-017-0867-9

47. Ullah, S., Khan, M.A., Farooq, M., Hammouch, Z., Baleanu, D.: A fractional model for the dynamics of tuberculosis infection using Caputo-Fabrizio derivative. Discrete Contin. Dyn. Syst., Ser. S 13(3), 975-993 (2020)

48. Ucar, E., Ozdemir, N., Altun, E.: Fractional order model of immune cells influenced by cancer cells. Math. Model. Nat. Phenom. 14(3), 308 (2019)

49. Samko, S.G., Kilbas, A.A., Marichev, O.I.: Fractional Integrals and Derivatives: Theory and Applications. Gordon \& Breach, Switzerland (1993)

50. Caputo, M., Fabrizio, M.: A new definition of fractional derivative without singular kernel. Prog. Fract. Differ. Appl. 1(2), $73-85$ (2015)

51. Wang, J., Zhou, Y., Medved, M.: Picard and weakly Picard operators technique for nonlinear differential equations in Banach spaces. J. Math. Anal. Appl. 389, 261-274 (2012)

52. Ullah, M.Z., Alzahrani, A.K., Baleanu, D.: An efficient numerical technique for a new fractional tuberculosis model with nonsingular derivative operator. J. Taibah Univ. Sci. 13(1), 1147-1157 (2019)

53. den Driessche, P.V., Watmough, J.: Reproduction numbers and subthreshold endemic equilibria for compartmental models of disease transmission. Math. Biosci. 180, 29-48 (2002)

54. Li, H., Cheng, J., Li, H.B., Zhong, S.M.: Stability analysis of a fractional-order linear system described by the Caputo-Fabrizio derivative. Mathematics 7(2), 200 (2019)

55. Li, C., Zeng, F.: The finite difference methods for fractional ordinary differential equations. Numer. Funct. Anal. Optim. 34(2), 149-179 (2013)

56. Jajarmi, A., Baleanu, D.: A new fractional analysis on the interaction of HIV with CD4+ T-cells. Chaos Solitons Fractals 113, 221-229 (2018). https://doi.org/10.1016/j.chaos.2018.06.009

\section{Submit your manuscript to a SpringerOpen ${ }^{\circ}$ journal and benefit from:}

- Convenient online submission

- Rigorous peer review

- Open access: articles freely available online

- High visibility within the field

Retaining the copyright to your article

Submit your next manuscript at $>$ springeropen.com 